\title{
Attention focuses on autism
}

$A_{p}$ Mons Month, an annual designation to help focus attention on autism for at least one month a year. But this year, several news stories and reports in peer-reviewed journals surrounding the highly contentious topic of whether vaccines have a role in the development of autism ensured heightened awareness of the disorder even before spring arrived.

Autism is a complex brain development disorder that impairs the development of social interaction and communication skills. Although it is known that abnormalities in the structure and/or function of the brain cause autism, the mechanisms initiating and underlying these abnormalities have been hard to pin down. Genetic factors have a crucial role, but no genes have been specifically linked to the disorder, and research indicates that environmental factors contribute. Determining the identity and precise role of the environmental factors is, however, proving controversial.

A study published in The Lancet (1) suggested, but did not prove, a link between the measles, mumps, and rubella (MMR) vaccination and autism. This led to a major health scare that resulted in a dramatic decrease in the proportion of children being given the MMR vaccination, in particular in the United Kingdom. Although the interpretation of the data published in The Lancet article was later retracted by most of the authors (2) and many subsequent studies have indicated no link between the MMR vaccination and autism $(3,4)$, a report this year has indicated that the proportion of children in the United Kingdom that received the MMR vaccination in 2007 was still way below that observed before 1998 and that this is largely because parents actively decide not to have their child immunized (5).

In parallel with the decrease in the proportion of children being given the MMR vaccination, the number of cases of measles in the United Kingdom has risen from 56 in 1998 to 971 in 2007, including one death. Although, unlike in the United Kingdom, no countrywide decrease in the MMR vaccination rate was observed in the United States, unusual outbreaks of measles have been reported recently. In one outbreak in California, the parents of 9 of the 12 children who became ill had chosen not to have their child vaccinated against measles and the other 3 children were too young to have received the vaccine. Although the reasons these parents chose not vaccinate their child against measles have not been revealed, another mother, whose child attends the same school as some of those who became ill, told the New York Times that she had chosen not to have her son vaccinated against measles because she believed that vaccines were linked to, among other things, neurological disorders (6).

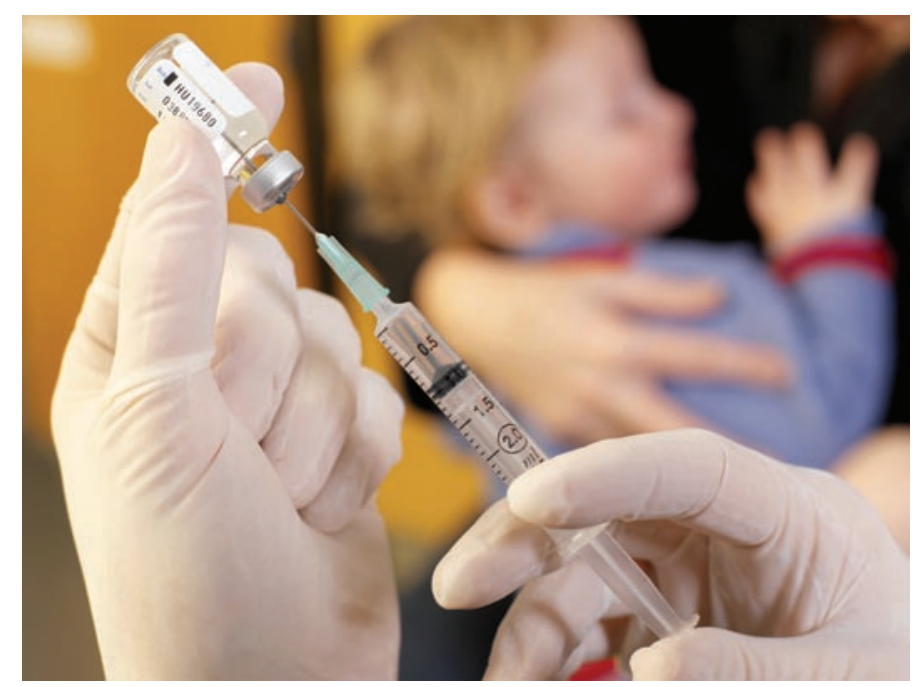

Although there is no scientific evidence to support the claim, some autism advocacy groups and parents believe that vaccines cause autism. Image credit: Photo Researchers Inc.

One of the issues is the heated debate that continues to rage about the role of the vaccine preservative thiomersal, which is a mercury-containing organic compound, in the development of autism. As described in a 2004 Institute of Medicine report (7), the balance of scientific evidence indicates that there is no link between exposure to thiomersal and autism. Despite this, vaccine manufacturers agreed with the Centers for Disease Control and Prevention (CDC) and the American Academy of Pediatrics in July 1999 that thiomersal should be reduced or eliminated in vaccines as a precautionary measure; although it is still to be found in most influenza vaccines. If, as some parents and autism advocacy groups still believe, there is a link between thiomersal and autism, it would have been expected that the number of children diagnosed with autism should have decreased since 1999. However, as reported recently for the state of California, the numbers have continued to rise (8).

The recent agreement by the US government to pay the family of a nine-year-old girl for injury caused by vaccines has only added fuel to the fire in the eyes of the many parents and autism advocacy groups that believe vaccines can cause autism. In the case, the girl's parents contended that five simultaneous vaccinations that she received as an infant either triggered or severely worsened her autism-like symptoms. Although the symptoms could have been triggered by a fever or infection, the CDC agreed to pay the family from a federal fund that compensates people injured by vaccines. As stated by Wendy Fournier, parent and president of the National Autism Association, many parents and autism advocacy groups see this ruling as a confirmation that "vaccines can and do cause children to regress into autism" (9). However, Julie Gerberding, head of the CDC, pointed out that "this does not represent anything other than a very special situation" (10). Indeed, the girl at the center of the story has a mitochondrial disorder, and as Edwin Trevathan, a pediatric neurologist who is Director of the CDC National Center on Birth Defects and Developmental Disabilities, said, "Most children with autism do not seem to have a mitochondrial problem, so this association ... is probably relatively rare" (10). 
Other experts agree that this ruling is unlikely to set a precedent for others seeking to link their child's autism to vaccinations they received as an infant, and Paul Offit, an expert in pediatric vaccinology at the University of Pennsylvania, was keen to highlight to the JCI that the CDC conceded this case without an evidentiary hearing, meaning that there was no burden of proof (i.e., no proof that the vaccines had caused the girl's autism).

As was noted to the JCI by Gerald Fischbach, Scientific Director of the Simons Foundation, an organization that seeks to fund research in basic sciences and mathematics, including autism research, "There is no epidemiological evidence of a link between vaccination and the development of autism, and it is exceedingly unwise not to have your child vaccinated, as the consequences of this can be devastating, not just for the unvaccinated child but also others in the community."

\section{Karen Honey}

1. Wakefield, A.J., et al. 2008. Ileal-lymphoid-nodular hyperplasia, non-specific colitis, and pervasive developmental disorders in children. Lancet. 351:637-641.

2. Murch, S.H., et al. 2004. Retraction of an interpretation. Lancet. 363:750.

3. Madsen, K.M., et al. 2002. A population-based study of measles, mumps, and rubella vaccination and autism. N. Engl. J. Med. 347:1477-1482.

4. Smeeth, L., et al. 2004. MMR vaccination and pervasive developmental disorders: a case-control study. Lancet. 364:963-969.

5. Pearce, A., et al. 2008. Factors associated with uptake of measles, mumps, and rubella vaccine
(MMR) and use of single antigen vaccines in a contemporary UK cohort: prospective cohort study. BMJ. doi:10.1136/bmj.39489.590671.25.

6. Steinhauer, J. 2008 March 21. Public health risk seen as parents reject vaccines. The New York Times. http://www.nytimes.com/2008/03/21/us/21vaccine. html?_r=2\&adxnnl=1\&oref=slogin $\&$ ad $x n n l x=12061$ 02260-a2PN8qo3OWaFOFPZZ9TXrw\&oref=slogin

7. Immunization Safety Review Committee. 2004. Immunization safety review: vaccines and autism. National Academies Press. Washington, DC, USA. 214 pp.

8. Schechter, R., and Grether, J.K. 2008. Continuing increases in autism reported to California's developmental services system: mercury in retrograde. Arch. Gen. Psychiatry. 65:19-24.

9. National Autism Association. 2008 February 28. Government concludes vaccines caused autism [press release]. http://www.nationalautismassociation. org/press022808.php.

10. The Associated Press. 2008 March 7. Vaccine settlement complex, may not be first. http://www.cnn. com/2008/HEALTH/conditions/03/07/autism. vaccines.analysis.ap/index.html. 